\title{
Application of Bayesian Networks for Learner Assessment in E-Learning Systems
}

\author{
S.R. Mangalwede \\ Department of Computer Science and Engineering, \\ Gogte Institute of Technology, \\ Belgaum, INDIA
}

\author{
Dr. D.H. Rao \\ Director, \\ MATS School of Business, \\ Belgaum, INDIA
}

\begin{abstract}
Traditionally, e-Learning content is delivered without taking the learner's traits into account. Content delivered to the learner should be personalized based on the learner profile so that learning can be effective. Also, assessment of a learner's learning objective is normally done by posing a set of questions without documenting the student's capabilities. A school of thought envisages assessing the real caliber of the student by posing questions that are linearly complex as the number of questions posed increase. This paper discusses the application of stochastic process model and Bayesian belief networks for learner assessment. The authors also discuss how it can be integrated into ongoing research into application of mobile agent technology in implementing case-based reasoning for content delivery in e-Learning systems. The implementation observations of such implementation vis-à-vis traditional assessment are also documented.
\end{abstract}

\section{Keywords}

Bayesian Networks, e-Learning, Mobile Agent, Stochastic Process.

\section{INTRODUCTION}

With the advent of internet and related technologies, e-Learning research related to personalization of content delivery and learner's assessment to gauge the progress have assumed significance. Though lot of interest has been generated in adaptive content delivery based on the profile of the learner, not much attention is given to the assessment of the learning objectives that in turn feed back into the upgradation of eLearning content[1-3]. Our ongoing research into the application of mobile agent technology for developing a framework for eLearning system attempts to use stochastic process model and Bayesian belief networks for learner assessment - one of the important components of e-Learning [4].

Learner assessment can be classified into three categories viz.,

1. Diagnostic assessment which is typically investigative, that is used to identify the current knowledge and skill level of the learner;

2. Formative assessment which can be used to determine the possible development activities required in order to improve the level of understanding; and
3. Summative assessment which is typically comprehensive, that is used to judge and grade a learner's level of understanding and meeting the learning objectives.

All the three forms of assessments are integrated into the proposed e-Learning framework and this paper specifically focuses on the application of the proposed approach for summative assessment.

The Bayesian networks are frequently used to model situations in which the causation plays a primal role, nevertheless this causation-the understanding of what is happening in certain moment, is not complete. It means that, for a given domain, a Bayesian network represents a combined probability distribution on a group of variables. A Bayesian network is a directed acyclic graph that maps the relationship called hypothesis-evidence. Bayesian networks are applied for industrial system diagnosis [5], medical diagnosis [6], for social science research [7].

This paper attempts to document our work of application of stochastic process model and Bayesian theory for learner assessment.

The rest of the paper is organized as follows: Section 2 introduces the stochastic process model and Bayesian belief networks. Section 3 discusses the model proposed by authors for learner assessment. In section 4, we discuss the implementation experiences and finally we conclude.

\section{STOCHASTIC PROCESS MODEL AND BAYESIAN BELIEF NETWORKS 2.1 Stochastic Process Model}

In probability theory, a stochastic process, or sometimes random process, is the counterpart to a deterministic process. Instead of dealing with only one possible reality of how the process might evolve under time, in a stochastic or random process there is some indeterminacy in its future evolution described by probability distributions. This means that even if the initial condition is known, there are many possibilities the process might go to, but some paths may be more probable and others less [8].

In the simplest possible cases such as stock market, a stochastic process amounts to a sequence of random variables known as a time series. Another basic type of a stochastic process is a random field like static images or random terrains, whose 
domain is a region of space, in other words, a random function whose arguments are drawn from a range of continuously changing values. One approach to stochastic processes treats them as functions of one or several deterministic inputs whose outputs are random variables: non-deterministic quantities which have certain probability distributions. Random variables corresponding to various times may be completely different. The main requirement is that these different random quantities all have the same type [9]. Although the random values of a stochastic process at different times may be independent random variables, in most commonly considered situations they exhibit complicated statistical correlations.

In mathematics, a stochastic matrix, probability matrix, or transition matrix is used to describe the transitions of a Markov chain. A stochastic matrix describes a Markov chain $X$ over a finite state space $S$.

If the probability of moving from $i$ to $j$ in one time step is $\operatorname{Pr}(j \mid i)$ $=P_{i, j}$, the stochastic matrix $P$ is given by using $P_{i, j}$ as the $i^{\text {th }}$ row and $j^{\text {th }}$ column element, e.g.,

$P=\left(\begin{array}{ccccc}p_{1,1} & p_{1,2} & \ldots & p_{1, j} & \ldots \\ p_{2,1} & p_{2,2} & \ldots & p_{2, j} & \ldots \\ \ldots & \ldots & \ddots & \ldots & \ddots \\ p_{i, 1} & p_{i, 2} & \ldots & p_{i, j} & \ldots \\ \ldots & \ldots & \ddots & \ldots & \ddots\end{array}\right] \quad---(1)$

Since the probability of transitioning from state $i$ to atleast one state $\mathrm{j}$ must be 1 , this matrix is a right stochastic matrix, so that

$$
\sum_{j} P_{i, j=1}
$$

The probability of transitioning from $i$ to $j$ in two steps is then given by the $(i, j)^{\text {th }}$ element of the square of $P$ :

$$
\left(P^{2}\right)_{i, j}
$$

In general, the probability transition of going from any state to another state in a finite Markov chain given by the matrix $P$ in $k$ steps is given by $P^{k}$.

\subsection{Bayesian Belief Networks}

The essence of the Bayesian approach is to provide a mathematical rule explaining how the existing beliefs should be changed in the light of new evidence. In other words, it allows scientists to combine new data with their existing knowledge or expertise. The canonical example is to imagine that a gifted newborn observes his first sunset, and wonders whether the sun will rise again or not. He assigns equal prior probabilities to both possible outcomes, and represents this by placing one white and one black marble into a bag. The following day, when the sun rises, the child places another white marble in the bag. The probability that a marble plucked randomly from the bag will be white (i.e., the child's degree of belief in future sunrises) has thus gone from a half to two-thirds. After sunrise the next day, the child adds another white marble, and the probability (and thus the degree of belief) goes from two-thirds to three-quarters. And so on. Gradually, the initial belief that the sun is just as likely as not to rise each morning is modified to become a near-certainty that the sun will always rise [10].

Mathematically, Bayes' rule states,

$$
\text { likelihood * prior }
$$

$$
\text { posterior = -------------------------- }
$$

or, in symbols,

$$
\mathrm{P}(\mathrm{R}=\mathrm{r} \mid \mathrm{e})=\mathrm{P}(\mathrm{e} \mid \mathrm{R}=\mathrm{r}) \mathrm{P}(\mathrm{R}=\mathrm{r})
$$

where, $\mathrm{P}(\mathrm{R}=\mathrm{r} \mid \mathrm{e})$ denotes the probability that random variable $\mathrm{R}$ has value $r$ given evidence e. The denominator is a normalizing constant that ensures the posterior adds up to 1 ; it can be computed by summing up the numerator over all possible values of R, i.e.,

$\mathrm{P}(\mathrm{e})=\mathrm{P}(\mathrm{R}=0, \mathrm{e})+\mathrm{P}(\mathrm{R}=1, \mathrm{e})+\ldots=$ sum_r $\mathrm{P}(\mathrm{e} \mid \mathrm{R}=\mathrm{r}) \mathrm{P}(\mathrm{R}=\mathrm{r})$

This is called the marginal likelihood (since we marginalize out over R), and gives the prior probability of the evidence.

\section{PROPOSED MODEL FOR LEARNER ASSESSMENT}

We have integrated the learner assessment into an e-Learning system that implements adaptive or personalized content delivery using case-based reasoning. The assessment module is implemented as follows: We have considered a set of questions for a course being offered by the e-Learning system. The set of questions are divided into 3 categories viz., Easy, Moderate and Difficult. Under each set of questions there are subsets. For e.g. in Easy category, we have developed questions that $\epsilon$ \{Easiest, Easier, Easy\}. Similarly questions are developed for the other two categories viz., Moderate and Difficult.

If we consider the subsets under each set as E11 for Easiest, E21 for Easier and E31 for Easy; similarly D11 for Slightly Difficult, D21 for Difficult and D31 for most difficult; similarly for Moderate set of questions, we have a right stochastic matrix that looks as shown in figure 1. 
These set of questions are stored in separate tables uniquely identified by the nature of questions. The proposed design model for summative assessment makes use of the Bayesian networks.

Bayesian Networks are compact networks of probabilities that capture the probabilistic relationship between variables, as well as historical information about their relationships [11]. They are very effective in modeling cases where some information is already known and incoming data is uncertain or partially unavailable.

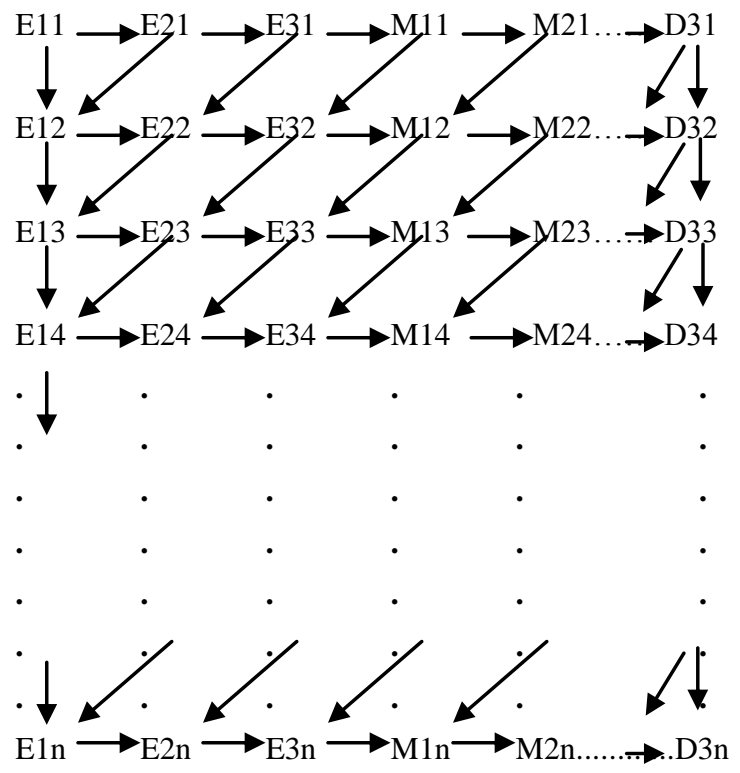

Figure 1. Schotastic Matrix of questions.

In our implementation, questions are structured in an acyclic graph that represents the relationship between two successive nodes (representing questions) as a causality relationship. When we consider two successive questions say $\mathrm{X}$ and $\mathrm{Y}$ with an edge connecting the two, we can have four sets of probabilistic relationships viz.,

1. Probability of answering $\mathrm{Y}$ correctly given that $\mathrm{X}$ is answered correctly,

2. Probability of answering $\mathrm{Y}$ incorrectly given that $\mathrm{X}$ is answered correctly,

3. Probability of answering $\mathrm{Y}$ correctly given that $\mathrm{X}$ is answered incorrectly, and

4. Probability of answering $\mathrm{Y}$ incorrectly given that $\mathrm{X}$ is answered incorrectly.

This priory and posteriori probabilistic relationship is captured using Bayesian networks. For this, we use class libraries of JavaBayes [12]. Figure 2 shows the Bayesian Network developed. Each node represents a question and the egde represents the transition from one question to another. The transition is done based on the conditional probabilities assigned as described above.
After the initial seed question (E11) is answered by the learner, the next question is posed based on the conditional probability of answering the next question. The next question chosen will have a lower probability of the learner answering correctly. The basic idea behind such an approach is to test the real understanding and the capability of the learner. When two or more questions have same probability, a question is chosen randomly among that set. The motivation is to never ask a question that the learner can answer effortlessly.

Each question will have an assigned grade score. This score will depend on the category to which the question belongs, with appropriate weightage attached to the category and sub-category to which the question belongs. A cumulative count is maintained that sums up the grade scores. At the end of the assessment, this count can be used to grade the student. Also, the profile of the learner, and the nature of content delivered during the process of learning along with the score determine the efficacy of the content.

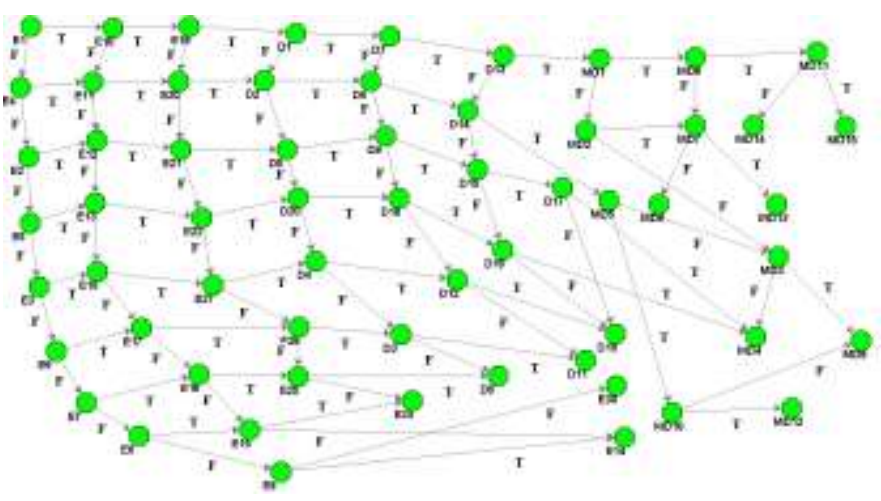

Figure 2. Bayesian Network of questions.

\section{IMPLEMENTATION EXPERIENCES}

In our implementation of e-Learning system, the learner database will profile the personal information about each learner, as well as information about their motivations, knowledge and skills, experience. The profile also accounts for the educational, cultural and technological background, preferences towards certain media for representation of the e-Learning content, learning performance, etc. Along with the profile, the system also conducts a diagnostic assessment to to ascertain the level of understanding of the learner as a prerequisite [13].

When a learner finishes a certain course/learning session, he/she will undergo self-assessment test (formative assessment) in order to check the understanding of his/her knowledge in that module. At that point, a mechanism for extracting the questions from learning materials and generating tests is activated. The results of those tests are used for updating the learner profile database. As previously stated the questions will be formative with each question being marked either as answered definitely, answered with hint or answer was prompted. The learner's responses will be recorded and this will be used for generating and delivering appropriate learning content next. 
In order to establish the efficacy of implementation of summative assessment, we took a random sample of learners with closely matching profiles and made a subset of them undergo the course content using the e-Learning system implemented in this work. Remaining subset of the learners was made to undergo the traditional e-Learning content (usually a set of static web pages that do not take the learner profile into account to personalize the content). After going through the course content in both the cases, the two sets of learners were made to undergo a summative assessment to evaluate the learner's understanding of the course.

Figure 3 shows the performance of the subset of learners who underwent the traditional e-Learning content. Figure 4 shows the performance of the remaining subset of learners whose profile was captured by the e-Learning system and content was delivered based on the learner profile. As can be observed from figure 5 that shows the performance comparison between the two sets of learners, a learner who gets content personalized based on his/her profile fares better in the assessment compared to the learner who goes through a static set of pages to meet the learning objectives.

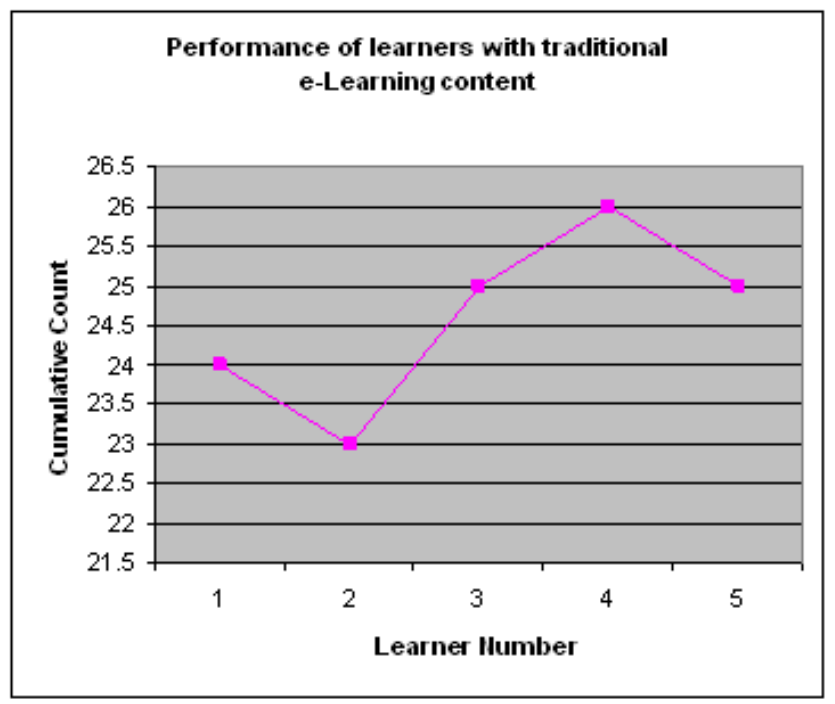

Figue 3. Performance of traditional e-Learers.

Since a learner's user context is normally subject to continuous change, the content must be selected as close as possible to the time when it is actually needed and on a per-learner basis [14].

The experiences justify the argument that achievement of learning objectives can be enhanced if the learning content is specifically personalized to individual learners' preferences, learning progress and requirements (captured as learner profile).

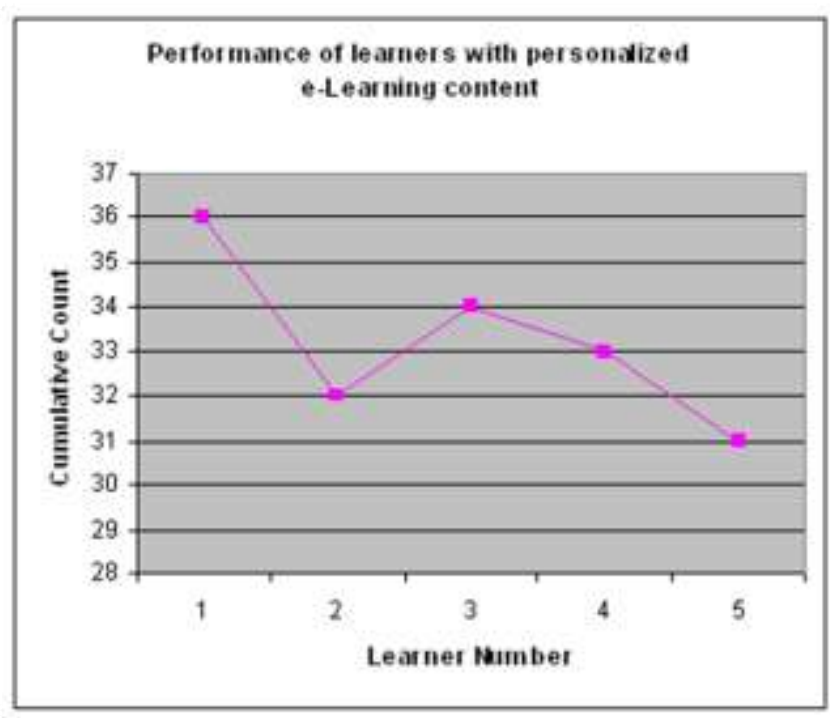

Figure 4. Performance of learners with personalization.

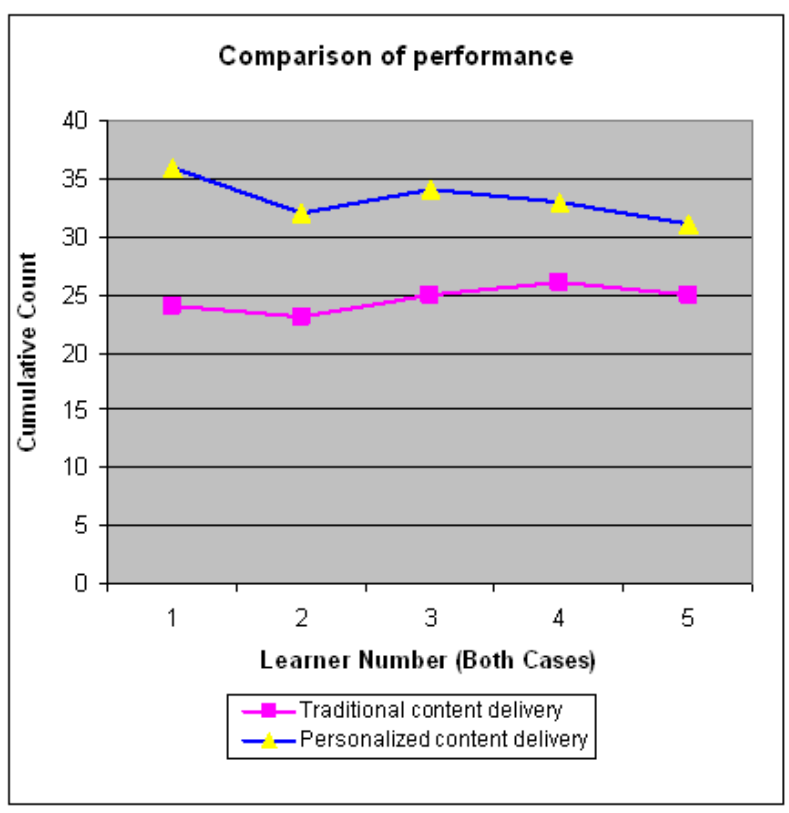

Figure 5. Comparison between the two cases.

\section{CONCLUSION}

There is a strong case in favor of personalization of e-Learning content delivered to the learner. Also, the approach to learner assessment may be looked from the viewpoint of assessing the learner capabilities by posing questions that the learner is deemed not to answer easily. Artificial (or computational) intelligence techniques such as Case-Based Reasoning, Stochastic Process Model and Bayesian Networks can provide for implementation of more effective e-Learning in the form of content delivery and learner assessment. Moreover, investigating suitable technologies to implement e-Learning systems is important as the learners are dispersed geographically. The proposed use of agent technology coupled with computational 
intelligence may be a feasible foundation for modeling eLearning systems.

\section{ACKNOWLEDGMENTS}

This work is supported in part by the Visvesvaraya Technological University, Belgaum, INDIA under Research Grant Scheme VTU/Aca-RGS/2008-2009/7196.

\section{REFERENCES}

[1] K.A. Papanikolaoui, M. Grigoriadou et al, Towards new forms of knowledge communication: the adaptive dimension of a web-based learning environment, Computers and Education 39, 4 (2002), 333-360.

[2] Hasan Al-Sakran, Developing E-Learning System Using Mobile Agent Technology, 0-7803-9521-2/06, 2006, IEEE.

[3] Tomasz Orzechowski, "The Use of Multi-Agents' Systems in E-Learning Platforms," Siberian Conference on Control and Communications SIBCON-2007, 1-4244-0346-4/07 2007 IEEE.

[4] S.R. Mangalwede and D.H. Rao, "Context-Aware Intelligent Multi-Agent Technology in Knowledge Grid Environments for E-Learning Systems," Proceedings of International Conference on Advances in Computing, Communication and Control, 2009. ICAC3'09. ACM SIGART, pp. 257 - 263. ISBN: 978-1-60558-351-8.

[5] Julio C. Ramírez V. et al., "Learning Bayesian Networks for Systems Diagnosis," Proceedings of the Electronics, Robotics and Automotive Mechanics Conference (CERMA'06).

[6] Peter Antal et al., Bayesian networks in ovarian cancer diagnosis: potentials and limitations, CBMSOO, Texas Medical Center, Houston, Texas, pp. 103-108.
[7] Sawhney N., "Bayesian Model Selection for Human Development Indicators," "A Hypertext History of Multiuser Dimensions," http://web.media.mit.edu/nitin/DevBayes/ DevBayes.pdf. 2001.

[8] Ramil' Salakhutdinov: Spinning inside the Ring Road (Kruzhenie v predelakh kol'tsevoi, 2006, reviewed by Greg Dolgopolov@ 2008.

[9] Papoulis, Athanasios \& Pillai, S. Unnikrishna (2001). Probability, Random Variables and Stochastic Processes. McGraw-Hill Science/Engineering/Math. ISBN 0-07281725-9.

[10] Kevin P. Murphy, "In praise of Bayes," http://www.cs.ubc.ca/ murphyk/Bayes/economist.html, 2000 .

[11] Christos E. Alexqkos, et al., "Integrating e-learning environments with computational intelligence assessment agents," Proceedings of World Academy of Science, Engineering and Technology, Volume 13, May 2006. ISSN: 1307-6884.

[12] Fabio Gagliardi Cozman, "JavaBayes, Bayesian Networks in Java," http://www.cs.cmu.edu/ javabayes/

[13] S.R. Mangalwede and D.H. Rao, "Case-Based Reasoning for adaptive content delivery in e-Learning systems using mobile sgents," Proceedings of International Conference on Contours of Computing Technology (Thinkquest-2010), pp. 49-54. Springer ISBN: 978-81-8489-988-2.

[14] Armin Ulbrich et al., "First steps towards personalization concepts in elearning," Lecture Notes in Informatics, GI, Gesellschaft für Informatik, Bonn. 2003. Pp. 229-233. Editors: Ulrich Reimer, Andreas Abecker, Steffen Staab, Gerd Stumme (eds.). 